\title{
Radar Imaging by Range Density Functions
}

\author{
Askin Demirkol ${ }^{1}$ and Erol Emre ${ }^{2}$ \\ 1 Department of Electrical and Computer Engineering,University of Missouri, Rolla,MO \\ 65409-0040 USA \\ demirkoleumr . edu \\ ${ }^{2}$ Department of Computer Engineering, Sakarya University, Sakarya-Turkey \\ emreesakarya.edu.tr
}

\begin{abstract}
In this paper, as an active sensor imaging technique, a new target density function in form of the range density function(RDF) is developed by Gabor transform which is called short time Fourier transform (STFT). It is shown that Gabor theory, (STFT) can be used as approach to imaging by active sensors by transmitting a waveform which is a kernel for this transform. Then an alternative signal dimension reduction approach is proposed to the developed technique by taking advantage of Walsh functions.

Keywords: active sensor imaging, SAR-ISAR, target density function, range density function, Gabor transform, Short time Fourier transform (STFT), Walsh function.
\end{abstract}

\section{Introduction}

Imaging is a mapping process from three dimensional object to two dimensional image $[1,2,3,4,5]$. This transformation is obtained by using signal transforms such as Fourier and Wavelet transforms $[1,6,7,2,3,4,5]$. Radar imaging is based on a multisensor image fusion technique, which is in the form of multiple-apertures and arrays $[8,9,10,11,12,13]$.

Target density function(TDF) is the reflectivity of spatially, continuously distributed targets and it is an important characteristic of radar imaging. TDF is known by different names such as ambiguity function, density function, target density function, object(target), object reflectivity function, doubly-spread reflectivity function, and reflection coefficient $[8,9,10,11,12,13]$.

There are two well known approaches on TDF. First one considers the integration of all point scatterers off the target scatterer centers. is able to obtain the whole object. This radar imaging technique is based on inverse Fourier transform(IFT) and used mostly in inverse synthetic aperture radar(SAR) studies $[1,16,2,3,4,5]$.

Second method on TDF is a dense target environment approach by Fowle and Naparst $[14,15]$. This takes into consideration the existence of densities of the targets in a high dense target environment. It is based on the ambiguity functions with two variables as range and velocity[17, 18, 19]. Especially, the advanced function in the dense target environment by Naparst is developed in a novel way. Rather than typical radar imaging, this is an approach to measure the closeness of the targets to each other 
in the dense target environment. However, in this work is inspired partly by Naparst model.

In this study, a new TDF is produced as a range density function considering a range-scanning angle plane different from the early approaches. This technique is developed based on Gabor theory which is called short term Fourier transform (STFT).

\section{Walsh Functions}

Walsh functions are orthogonal functions and composed of square waves with (0-1) amplitudes. Unlike the Rademacher functions, Walsh functions are complete. Mathematical theory of Walsh functions corresponds to Fourier analysis-based sine-cosine functions $[20,21,22,23]$.

Walsh functions are defined in a limited time interval, $T$, known as the time-base. Like the sine-cosine functions, two entities are required for a complete definition. These are a time period, $t$, which is normalized to the time base as $t / T$, and an ordering number, $n$, which is related to frequency. $\mathrm{A}$ Walsh basis function is represented by $W a l(n, t)$. A general Walsh function with pulse basis functions can be written as $[20,21,22,23]$

$$
W a l(n, t)=\operatorname{sign}\left[(\sin 2 \pi t)^{b_{0}} \prod_{k=1}^{m}\left(\cos 2^{k} \pi t\right)^{b_{k}}\right]
$$

where $n$ and $m$ are related to each other. If $u$ is a binary value of the decimal, $n$, and $g(u)$ is a number of digits, then $m$ is represented as

$$
m=g(u)-1
$$

$b_{0}$ and $b_{k}$ in Equation 1 are either 0 or 1.

A set of Walsh functions derived from Equation 1 is given in Figure 1 [20, 21, 22, 23]. These sets of Walsh functions are in form of typical radar pulse train.

While behavior of both Fourier and Walsh series are similar, basis functions have different forms. Walsh function can be expressed as a time series similar to the Fourier theory:

$$
f(t)=\sum_{k=0}^{\infty} F_{k} W a l(k, t)
$$

If this is compared with Fourier series, $p(t)=\sum_{k=-\infty}^{\infty} \alpha_{k} e^{j k \omega_{0} t}$, their basis functions become important separators. The basis functions are infinite in Fourier series $(-\infty \leq$ $k \leq \infty)$, while finite in Walsh series $(0 \leq k \leq \infty)$. The finite basis functions provide important advantage in signal processing in terms of dimension reduction.

Two new functions, sal and cal, which are analogues of sine and cosine functions in Fourier series, are defined by Walsh functions $[20,21,22,23]$.

$$
f(t)=a_{0} \operatorname{Wal}(0, t)+\sum_{k=1}^{\infty}\left[a_{k} \operatorname{cal}(k, t)+b_{k} \operatorname{sal}(k, t)\right]
$$




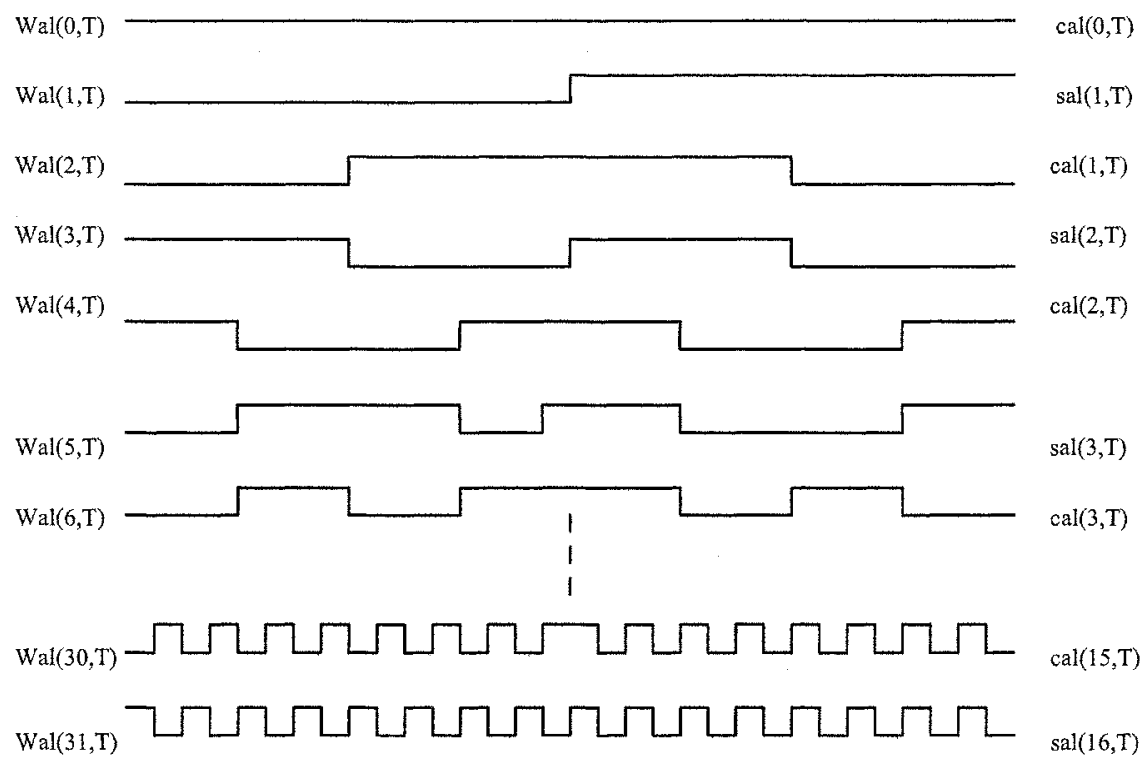

Fig. 1. A set of Walsh functions.

where

$a_{0}=\int_{-1 / 2}^{1 / 2} f(t) \operatorname{Wal}(0, t) d t, a_{k}=\int_{-1 / 2}^{1 / 2} f(t) \operatorname{cal}(k, t) d t, b_{k}=\int_{-1 / 2}^{1 / 2} f(t) \operatorname{sal}(k, t) d t$

\section{Imaging by Gabor Theory}

In this paper, an active sensor imaging is studied by an alternative target density function(TDF), which is based on a linear phased array radar system and the rangescanning angle. New target density function, $g(R, \beta)$ is composed of two variables, which are the range $R$, and the scanning angle $\beta$. Definition of $g(R, \beta)$ in developed here is given as the following.

Definition 1. Target Density Function is the limit of the ratio of the amplitude of the signal reflected from an infinitesimally neighborhood about the point $(R, \beta)$ to the amplitude of the incoming signal.

By this definition, the new target density function $g(R, \beta)$ is;

$$
g(R, \beta)=\lim _{d(\Omega) \rightarrow 0} \frac{A_{r}}{A_{t}}
$$

where $d(\Omega)$ is the diameter of the disc about the point $(R, \beta) \in \Omega, A_{r}$ and $A_{t}$ are the amplitudes of the reflected and the transmitted signals, respectively. 
In this definition, the target density function(TDF) is relevant to the the reflectivity of spatially, continuously distributed targets. This approach is different from the conventional target density function definitions stated early. Instead of ambiguity functions based on range-velocity variables, the imaging is taken by a new target density function with the range and scanning angle.

Let us consider the target plane shown in Figure 2, where $\beta$ is $\cos \theta$ and $R$ is the range from the target to the radar, and the sensor elements in the linear phased array radar system are located equally. As seen in Figure 2, the target density function is a

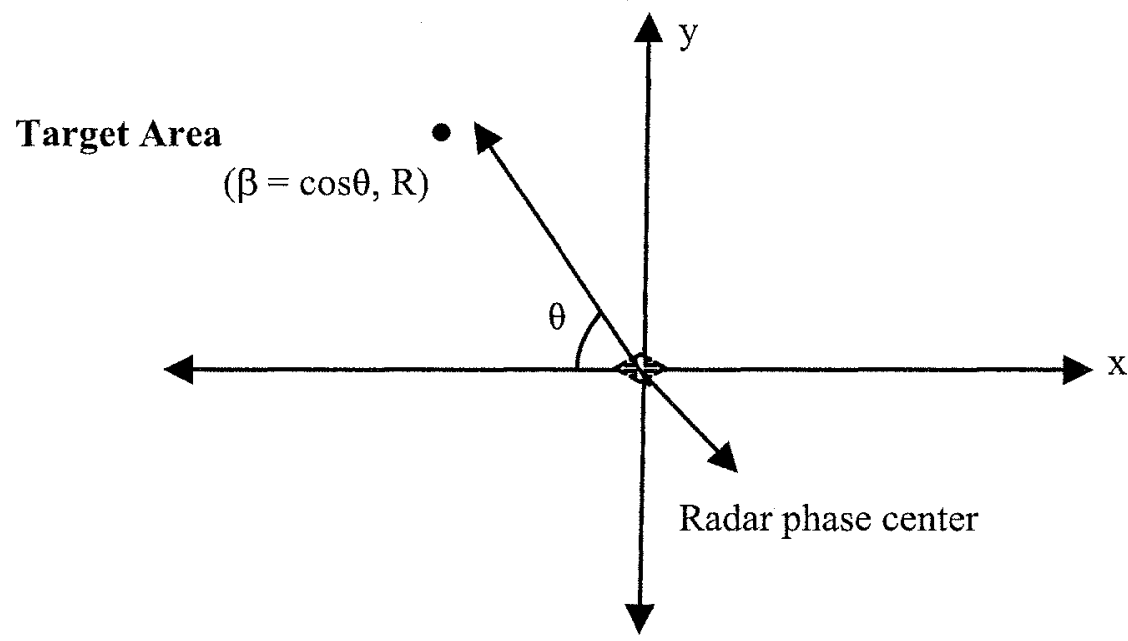

Fig. 2. Radar-Target Coordinate system.

function of the spatial coordinates $(R, \beta)$ in the upper semi-plane.

Now, let us obtain the target density function. Let $P(t)$ be any periodic function of time, such as a train of pulses, where

$$
\begin{gathered}
p(t)=\sum_{k=-\infty}^{\infty} \alpha_{k} e^{j k \omega_{0} t} \\
\omega_{0}=2 \pi \times \mathrm{PRF}
\end{gathered}
$$

where PRF is the pulse repetition frequency.

$$
W(t)=e^{j \omega_{c} t}
$$

Where $W(t)$ is the carrier signal.

$$
s_{m}(t)=p(t) W(t)
$$


Where $s_{m}(t)$ is the modulated signal transmitted in the fixed direction $\beta$, which can be done by a directed single antenna or a phased array using beamforming.

The reflectivity of one point at $g(R, \beta)$

$$
y(t, \omega)=s_{m}(t-2 R / c-\beta x / c) g(R, \beta)
$$

Let us generalize Equation 11 for the whole radar-target semi upper plane by superpositioning principle considering all point scatterers related to the range-angle.

If $g(R, \beta)$ is the reflectivity of the point $(R, \beta)$, and $R_{1}$ is the maximum range of interest target area; then the total reflected incoming signal to the phase center will be

$$
\begin{aligned}
y(t, \omega) & =\int_{-1}^{1} \int_{0}^{R_{1}} s_{m}\left(t-\left(\frac{2 R+\beta x}{c}\right)\right) g(R, \beta) d R d \beta \\
& =\int_{-1}^{1} \int_{0}^{R_{1}} p\left(t-\left(\frac{2 R+\beta x}{c}\right)\right) W\left(t-\left(\frac{2 R+\beta x}{c}\right)\right) g(R, \beta) d R d \beta
\end{aligned}
$$

where $y(t, \omega)$ is the output of the sensor located at center (the feature space), and $c$ is the speed of light.

If Equation 7 substitutes in Equation 12, the total reflected incoming signal to the phase center will be,

$$
y\left(t, \omega_{0}\right)=\int_{-1}^{1} \int_{0}^{R_{1}} \sum_{k=-\infty}^{\infty} \alpha_{k} e^{j k \omega_{0}\left(t-\left(\frac{2 R+\beta x}{c}\right)\right)} W\left(t-\left(\frac{2 R+\beta x}{c}\right)\right) g(R, \beta) d R d \beta
$$

Now a new target density function is defined by utilizing Definition 1 and Figure 2 . This function is the range density function $(R D F)$.

In a linear phased array of point sensor system, if $R$ is the range from the sensor in a fixed direction $(\beta)$, which is direction cosine of the line joining the point and the phase center, as a new target density function, the new range density function (RDF) is defined as follows. $R$.

Definition 2. Range Density Function, $f(R)$ is the reflectivity of the point at range

By this definition, $f(R)$ represents the image along the range or the distance to the sensor.

Let us formulate this definition. The range density function function $f(R)$ or $g_{\beta}(R)$ at a fixed angle, $\beta$,

$$
f(R) \equiv g_{\beta}(R) \equiv g(\beta, R)
$$
be

By the definition of the point at a fixed angle, $\beta$ and Equation 14, Equation 13 will

$$
y\left(t, \omega_{0}\right)=\int_{0}^{\infty} \sum_{k=-\infty}^{\infty} \alpha_{k} e^{j k \omega_{0}\left(t-\frac{2 R}{c}\right)} W(t-2 R / c) f(R) d R
$$

Let's define

$$
t_{\perp}=\frac{2 R}{c} \text { and }
$$




$$
W(t)=W_{\perp}(-t)
$$

and uniquely define $h$ to be the function such that

$$
f(R)=h\left(t_{\perp}\right)
$$

Then Equation 15 can be rewritten as

$$
y\left(t, \omega_{0}\right)=\frac{c}{2} \sum_{k=-\infty}^{\infty} \alpha_{k} e^{j k \omega_{0} t} \int_{0}^{\infty} e^{-j k \omega_{0} t_{\perp}} W\left(t_{\perp}-t\right) h\left(t_{\perp}\right) d t_{\perp}
$$

If Equation 19 is demodulated by $\frac{2}{c} \frac{1}{\alpha} e^{-j k \omega_{0} t}$

$$
y_{k}\left(t, \omega_{0}\right)=\int_{0}^{\infty} e^{-j k \omega_{0} t_{\perp}} W\left(t_{\perp}-t\right) h\left(t_{\perp}\right) d t_{\perp}
$$

Extend the domain of definition of $h\left(t_{\perp}\right)$ to $(-\infty, \infty)$ by defining $h\left(t_{\perp}\right) \equiv 0$ for $t_{\perp}<$ 0 . Then, it is seen that Equation 20 is the Gabor transform, which is known short-time Fourier transform, STFT of $h\left(t_{\perp}\right)$, that is $y_{k}\left(t, \omega_{0}\right)$ evaluated at the frequency $\omega_{0}$.

The inverse Gabor (STFT) transform yields $h\left(t_{\perp}\right)$ as

$$
h\left(t_{\perp}\right)=\frac{1}{2 \pi\|W\|^{2}} \int_{-\infty}^{\infty} \int_{-\infty}^{\infty} y_{k}\left(t, \omega_{0}\right) e^{j k \omega_{0} t_{\perp}} W^{*}\left(t_{\perp}-t\right) d \omega_{0} d t
$$

which is desired result. One can obtain the reflectivity of a target area at angle $\beta$, by transmitting $e^{j k \omega_{0} t} W(t)$ with varying $\omega_{0}$, and observing the reflected signal for all time. In theory, if the Gabor window function is chosen plausible as a band-limited function, $y_{k}\left(t, \omega_{0}\right)$ can be determined from its values over any finite length time interval, and the target density function $h\left(t_{\perp}\right)$, can be recovered[24]. Thus, by using a novel target density function $f(R)$ or $g_{\beta}(R)$, the radar targets can be imaged by utilizing Short Time Fourier Transform.

Infinity of $k$ in Equation 21 can be optimized by some filtration, compressing or estimation methods. However, an alternative way may be proposed to reduce $k$ dimension and smooth the new TDF;

- Walsh Approach: While the new TDF is developed, at the beginning, the basis functions of the modulating signal had infinite dimensions. In contrast to infinite basis functions $(-\infty \leq k \leq \infty)$ in the Equation 7, Walsh functions are expressed in finite pulse basis functions in Equation 3. They have an essential advantage to the radar imaging in terms of basis dimension reduction.

In case of using Walsh functions with pulse form, this function in the Equation (3) will replace the Equation 7 in the new algorithm as a modulating Walsh function with finite dimensions. The Walsh function in question is a modulating signal in the form of a pulse train. After Walsh function is chosen with respect to some parameters like $P R F$ in Equation (7), the new algorithm can resume the remaining steps after Equation (8) in a similar manner. 


\section{Summary and Conclusion}

In this paper, an alternative target density function(TDF) is obtained by a new algorithm and technique differently from the conventional approaches. Main contributions of this study ;

- A proposed target density function algorithm: The target density function (TDF) is represented in form of the range density function considering a novel range and scanning angle plane. It is produced by a new technique based on Gabor or short time fourier transform (STFT). It is shown that Gabor theory (STFT) can be used as approach to imaging by active sensors by transmitting a waveform which is a kernel for this transform such as a window function.

The present TDF is generated partly by analogy to Fowle-Naparst and SAR-ISAR approaches.

- Comparing to Fowle-Naparst: As an advanced work of Fowle, Naparst target density function is developed for a high dense target environment with multiple targets, whose velocities are close to each other. This TDF acts like a separator rather than an imaging function for the targets at the distance with a given velocity.

TDF proposed here is obtained by a scanning angle and range in a high dense target environment. The main difference is in the imaging approach, which is capable of sensor imaging the targets in a dense target environment via phased array radar system.

- Comparing to ISAR: While ISAR imaging is based on multi-aperture principle, the present imaging method is a multi-sensor image fusion technique based on the phased array radar system. On the contrary, the proposed target density function is produced by the integration of scanning angles at a fixed range.

\section{References}

1. Chen, V.C., Ling, H.: Time-Frequency transforms for radar imaging and signal analysis, (2002)

2. Gupta, I.J.: "High-Resolution radar imaging using 2-D linear prediction," IEEE Transactions on antennas and propagation, 42 January (1994) 31-37

3. Odendaal, J.W.: "2-D Radar Imaging," Communications and Signal Processing, 1994. COMSIG-94., Proceedings of the 1994 IEEE South African Symposium on, 4 October (1994) 146-151

4. Prickett, M.J.: "Principles of inverse synthetic aperture radar(ISAR) imaging," IEEE EASCON, (1980), 340-344

5. Ausherman, D.A., Kozma, A., Walker, J., Jones, H.M., Poggio, E.C.: "Developments in radar imaging," IEEE Transactions on Aerospace and Electronic Systems 20 no.4 (1984) $363-400$

6. Chen, V.C. Qian, S.: "Time frequency transform vs. fourier transform for radar imaging," Time-Frequency and Time-Scale Analysis, 1996., Proceedings of the IREE-SP International Symposium on, 18-21 June (1996) 389-392 
7. Krone, A.W., Munson, D.C.: “A Fourier model of ISAR imaging of approaching targets," Acoustics, Speech, and Signal Processing, 1992. ICASSP-92., 1992 IEEE International Conference on, 3 23-26 March (1992) 13-16

8. Wald, L.: "Some terms of reference in data fusion" IEEE Transactions on geoscience and remote sensing, 37, no.3, May (1999) 1190-1193

9. Sarma, V.V.S., Raju, S.: "Multi-sensor data fusion and decision support for airborne target identification," IEEE Transactions on systems, man, and cybernetics, 21 no.5 (1991)

10. Zhou, Y.T.: "Multi-sensor image fusion," Image Processing, 1994. Proceedings. ICIP-94., IEEE International Conference, 1 13-16 November (1994) 193-197,.

11. Durrant-Whyte, H.F.: "elements of sensor fusion," Intelligent Control, IEE Colloquium on, 19 Febuary (1991) 5/1-5/2

12. Varshney, P.K.: "multi-sensor data fusion," electronics and communication engineering journal, December (1997)

13. Hall, D.L., Llinas, J.: "An Introduction to multi-sensor data fusion," Proceedings of the IEEE , 85 Issue.1, January (1997) 6-23

14. Fowle, E.N., Kelly, E.J., Sheehan, J.A.: "Radar system performance in a dense-target environment," IRE Int.Convention record, no.4 (1961) 136-145

15. Naparst, H.: "Dense target signal processing," IEEE Transactions on information theory 37 no.2 March (1991)

16. Siebert, W.McC.: "A radar detection philosophy," IEEE Transactions on Information Theory, 2 Issue. 3 September (1956) 204-221

17. Woodward, P.M.: Probability and information theory with applications to radar,(1957).

18. Blahut, R.E., Wilcox, C.H., Miller, W.: "The synthesis problem for radar ambiguity functions," Springer-Verlag, Mathematics subject classifications:78A45,22E70,43A80,(1991) 229-260

19. Auslander, L., Tolimeri, R.: "Radar ambiguity functions and group theory," SIAM,J.Math.Anal. 16 (1985) 577-601

20. Walsh, J.L.: A closed set of normal orthogonal functions. Amer. J. Math. 45 (1923) 5-24

21. Beauchamp, K.G.: Walsh functions and their applications. Academic press, (1975)

22. Tzafestas, S.G.: Walsh functions in signal and systems analysis and design. A Hutchinson Ross Publication (1985).

23. Harmuth, H.F.: Applications of Walsh functions in communications. IREE Spectrum November (1969) 8-2-91

24. Papoulis, A.: "A new algorithm in spectra analysis and band-limited extrapolation," IREE Transactions in Circuits and Systems, v.CAS-22, September (1975) 735-742 